\title{
ESTANDARIZACIÓN DE LA PRELIMPIEZA DEL JUGO DE CAÑA DE AZÚCAR PARA LAS ESTANCIAS PANELERAS DE SAN MARCOS-SUCRE
}

\author{
"STANDARDIZATION OF THE PRE CLEANING OF SUGAR CANE \\ JUICE FOR FARMS SUGARCANE SAN MARCOS-SUCRE"
}

\author{
Adolfo Javier De Oro Torres, ${ }^{1}$ Germán José Narváez, Gómez ${ }^{2}$
}

\begin{abstract}
RESUMEN
La producción de panela en Colombia es de 1'587.893 Ton/año, que representaron 538.905 millones de pesos en 2002, cifra que convierte al país en el segundo productor mundial. El cambio de hábitos alimenticios y el estilo de vida de la población, así como la rápida evolución social y tecnológica que viene experimentando la humanidad, representan una amenaza para la supervivencia de esta agroindustria, la cual se encuentra todavía en una etapa primaria de desarrollo, a pesar de ser la base de la economía de varias regiones del territorio colombiano. Las nuevas tendencias de la sociedad hacia una alimentación natural han creado un mercado potencial. Se espera, entonces, que esta investigación se convierta en una herramienta importante para mantener e incrementar el consumo de panela tradicional, mejorando aspectos importantes en la prelimpieza de jugos, ya que una de las razones por las cuales el proceso de producción es lento, es la deficiencia que existe en el envío de un jugo limpio a la cocción. Con este propósito se caracterizó el jugo de caña y se realizaron pruebas con modelos de prelimpiadores establecidos por el CIMPA (Centro para la Investigación y el Mejoramiento de la Agroindustria Panelera) para medir la velocidad de pre limpieza y escoger el mejor diseño. La información fue tabulada y utilizada para aplicar un diseño experimental que ayudó a interpretar todos los datos y a llegar a las conclusiones y recomendaciones que se presentan en este trabajo.
\end{abstract}

Palabras clave: pre limpieza de jugos, caña de azúcar, calidad, panela tradicional, agroindustria.

\begin{abstract}
Panela production in Colombia is 1'587.893 tons / year, which represents 538.905 million pesos for 2002, figures that make this country the second largest producer of panela. Changing eating habits and lifestyle of the population, and the rapid social and technological

1 Ingeniero Agroindustrial, Especialista en Ingeniería de Procesos en Alimentos y Biomateriales. UNAD. Universidad de Sucre, Sincelejo - Sucre. E-mail: adolfodeoro@yahoo.es.

2 Ingeniero Agroindustrial, Especialista en Ingeniería de Procesos en Alimentos y Biomateriales. UNAD. Universidad de Sucre, Sincelejo- Sucre. E-mail: gejonago@yahoo.es.
\end{abstract}


developments that humanity is experiencing, are a threat to the survival of this agro-industry, which is still at an early stage of development, despite being the backbone of the economy of several regions of Colombia. The new trends in society towards natural food have created a potential market for the agroindustry. To this end it is hoped that this research may become an important tool to maintain and increase the consumption of traditional sugar cane, improving important aspects of the pre juice as one of the reasons the process of Panela production is slow, due to the deficiency that exists to send a clean cooking juice. For this purpose cane juice characterized and tested with models established by the CIMPA precleaning (Centre for Research and Improvement panelera Agribusiness) to measure its speed precleaning and choose the best design. The information was tabulated and used to apply an experimental design that helped to interpret all the data, reaching conclusions and recommendations of this research.

Key words: precleaning juice, sugarcane, quality, traditional panela, agro industry.

Aceptado: mayo 10 de 2009

Aprobado: mayo 26 de 2009

\section{INTRODUCCION}

La agroindustria de la panela en Colombia se destaca por su producción y consumo. Colombia ocupa el primer lugar en consumo, con una cifra de 31,2 Kg/Hab al año, cifra que supera de lejos al promedio mundial $(2,1 \mathrm{Kg} / \mathrm{Hab})$ y al mayor productor mundial, la India, cuyo consumo fue de $8,4 \mathrm{Kg} / \mathrm{Hab}$. Se estima que el crecimiento anual de la panela es del $1.7 \%$, lo que la convierte en uno de los productos más promisorios de los próximos años [2] [6].

El consumo de la panela se explica por el comportamiento de la producción, en la medida en que no hay importaciones y las exportaciones son marginales. Durante la última década, la producción creció en menor proporción al crecimiento vegetativo de la población, lo que generó una reducción en consumo como resultado de la competencia del azúcar (tanto como edulcorante como en forma de panela, azúcar derretida), los edulcorantes sintéticos y las bebidas artificiales [13]. Una de las actividades dentro del proceso productivo de la panela, que asegura la calidad del producto final, es la efectividad en la etapa de prelimpieza. En la medida que se obtiene un jugo de caña de azúcar libre de bagacillos, sólidos insolubles e impurezas el proceso se hace más eficiente. El propósito de la prelimpieza no es mejorar la clarificación, sino enviar un jugo limpio a la paila de clarificación, para así, tener un proceso de limpieza y una concentración de jugos más rápidos [4].

\section{ANTECEDENTES}

Una de las zonas productoras de panela más importantes del departamento de Sucre, es el municipio de San Marcos. El número de variedades de caña panelera existente, convierte a la región en una zona apta para incursionar tanto en el ámbito micro empresarial, como en mercados nacionales e internacionales. El 100\% de los trapiches en la zona de estudio, se 
caracteriza por su débil producción e infraestructuras y equipos inadecuados [12]. Aunque la mayoría de los cañicultores posee molinos semi industriales, los procesos de limpieza, cocción, batido y moldeo siguen siendo deficientes, sin tener en cuenta las Buenas Prácticas de Manufactura (BPM). La ineficiencia en los procesos hace que la comercialización sea pobre; hasta ahora solo se comercializa en la región y los intentos por irrumpir en mercados más exigentes han sido fallidos [3].

Uno de los problemas que más afecta el tiempo de residencia en el proceso de clarificación, es la condición del jugo de caña cuando entra a la paila de clarificación. El jugo sale del molino con restos de bagazo, tierra y lodo, los cuales aumentan la temperatura de ebullición y, por ende, el proceso de limpieza tarda demasiado. En varias zonas de Colombia [8] ya se ha implementado el uso de prelimpiadores para disminuir los tiempos de clarificación. Los resultados han sido satisfactorios, a tal punto, que se obtienen jugos más limpios y claros y se reduce el tiempo del proceso. El funcionamiento de los equipos de madera es igual que el de los de acero inoxidable, aunque existe un riesgo para el consumidor, pues la calidad se ve comprometida [1]. La diferencia radica en la duración del material, mayor facilidad en la limpieza y, lo más importante, en la higiene que tiene relación con las Buenas Prácticas de Manufactura. Lo ideal sería trabajar con material de acero inoxidable. Sin embargo, son muy costosos para las personas de la zona, por lo que es necesario buscar otras alternativas que den solución al problema [9].

\section{METODOLOGÍA}

Para el desarrollo de este proyecto, se realizó un estudio de carácter investigativo en las estancias paneleras de los Garridos, los Arrieta y los Hoyos, en las veredas de Candelaria y Monte Grande en el Municipio de San Marcos-Sucre. Toda la información necesaria para construir los prelimpiadores se obtuvo por medio de consultas bibliográficas, revistas y la Internet. Así mismo, gracias al apoyo y asesoría de entidades con experiencia en el tema, como el Centro para la Investigación y Mejoramiento de la Agroindustria Panelera-CIMPA y la Fundación Gaviota ubicada en la ciudad de Sincelejo, Sucre-Colombia.

\section{Caracterización de los jugos}

Se comenzó con la caracterización del jugo de caña de azúcar para establecer su calidad en el momento que sale del molino. De este modo, se pudo conocer la cantidad de residuos, su densidad y contenido de sólidos solubles.

\section{Consulta bibliográfica}

Se hizo revisión de la bibliografía seleccionada, para observar los diferentes diseños de prelimpiadores recomendados por el CIMPA y los trapiches paneleros reconocidos en el país. Además, se revisaron algunas revistas publicadas por CORPOICA y el CIMPA. 


\section{Adecuación de diseño}

Una vez conocido el diseño del pre limpiador, éste se ajustó a la capacidad de la molienda del trapiche. Las dimensiones fueron establecidas para trapiches con capacidad de $0.25 \mathrm{Ton} / \mathrm{h}$ hasta 1 Ton/h [11]. Las masas del molino se ajustaron adecuadamente para reducir la salida de sólidos en el jugo.

\section{Pruebas de velocidad de prelimpieza}

Se construyeron tres modelos demostrativos de pre limpiadores. Se utilizó la madera para su elaboración con el propósito de determinar la eficiencia de cada uno. Cada modelo tenía medidas diferentes, para estandarizar el mejor. En el proceso de selección se tuvieron en cuenta como variables de medición, la cantidad de litros de jugo por minuto (Litros/min) que lograba limpiar el pre limpiador y la densidad del jugo ( $\mathrm{Kg} / \mathrm{Litro})$, la cual depende de la cantidad de sólidos que este posea. Se repitió el proceso cuatro veces con cada pre limpiador y se realizó un diseño de experimentos con un ANOVA (análisis combinado con diseño completamente al azar, 3 tratamientos con 4 repeticiones), así como una comparación de tratamientos por contrastes ortogonales. Finalizados los análisis estadísticos, se escogió el diseño más eficiente.

\section{Tiempo de llenado de los prelimpiadores}

El tiempo de llenado de los prelimpiadores se determinó estableciendo las dimensiones, las alturas de las aberturas de carga y descarga y el ángulo de las pendientes dentro de los mismos, así como el caudal de jugo proveniente de los trapiches. Lo anterior se tuvo en cuenta, con el fin de establecer criterios de comparación (utilizando el teorema del transporte de Reynolds) donde se tiene un sistema y un volumen de control fijo e indeformable que coinciden en un instante dado [5].

\section{RESULTADOS Y ANÁLISIS}

\section{Análisis estadísticos}

Los resultados de las pruebas se muestran en la siguiente tabla. En esta se observa el promedio de velocidades en las tres zonas, expresando la velocidad de prelimpieza en litros por minuto:

Tabla 1. Velocidad de pre limpieza (Litros/min)

\begin{tabular}{|l|l|l|l|}
\hline Pre limpiador & 1 & 2 & 3 \\
\hline
\end{tabular}




\begin{tabular}{|c|c|c|c|}
\hline Repetición & $\begin{array}{l}\text { Largo*Alto*Ancho } \\
(0.8 \mathrm{~m} \times 0.3 \mathrm{mx} 0.15 \mathrm{~m})\end{array}$ & $\begin{array}{l}\text { Largo*Alto*Ancho } \\
(1.1 \mathrm{mx} 0.3 \mathrm{mx} 0.15 \mathrm{~m})\end{array}$ & $\begin{array}{c}\text { Largo*Alto*Ancho } \\
(0.8 \mathrm{mx} 0.3 \mathrm{mx} 0.3 \mathrm{~m})\end{array}$ \\
\hline 1 & 2.29 & 3.33 & 3.42 \\
\hline 2 & 2.18 & 3.03 & 4.22 \\
\hline 3 & 2.43 & 3.28 & 3.37 \\
\hline 4 & 2.32 & 3.1 & 4.13 \\
\hline Promedio & 2.3 & 3.2 & 3.8 \\
\hline
\end{tabular}

Fuente: de oro, Narvaez 2008.

A estos datos se les aplicó un análisis de varianza ANOVA para determinar si existía diferencia entre los prelimpiadores. Los resultados fueron:

Tabla 2. Análisis de varianza (ANOVA)

\begin{tabular}{|c|c|c|c|c|c|c|}
\hline Grupos & Cuenta & Suma & Promedio & Varianza & & \\
\hline Pre limpiador 1 & 4 & 9.22 & 2.305 & 0.01056667 & & \\
\hline Pre limpiador 2 & 4 & 12.74 & 3.185 & 0.02043333 & & \\
\hline Pre limpiador 3 & 4 & 15.14 & 3.785 & 0.20456667 & & \\
\hline $\begin{array}{c}\text { Origen de las } \\
\text { variaciones }\end{array}$ & $\begin{array}{l}\text { Suma de } \\
\text { cuadrados }\end{array}$ & $\begin{array}{l}\text { Grados de } \\
\text { libertad }\end{array}$ & $\begin{array}{c}\text { Promedio } \\
\text { de los } \\
\text { cuadrados }\end{array}$ & $\mathbf{F}$ & Probabilidad & $\begin{array}{c}\text { Valor } \\
\text { crítico } \\
\text { para } \mathbf{F}\end{array}$ \\
\hline Tratamientos & 4.433066667 & 2 & 2.216533333 & $28.2281024 *$ & 0.00013253 & 8.02151731 \\
\hline Error & 0.7067 & 9 & 0.078522222 & & & \\
\hline Total & 5.139766667 & 11 & & & & \\
\hline
\end{tabular}

* Existe diferencia significativa entre los tratamientos.

Fuente: de oro, Narvaez 2008.

Con base en los resultados del análisis de varianza, podemos darnos cuenta que existe diferencia entre los pre limpiadores, por lo que se establecieron hipótesis para determinar cuál de ellos era el mejor. El nivel de significancia fue del 1\%.

1) Que los tratamientos 1 y 2 son iguales al tratamiento 3

$\left(\mathrm{U}_{1}+\mathrm{U}_{2}\right) / 2=\mathrm{U}_{3}$

$\mathrm{U}_{1}+\mathrm{U}_{2}-2 \mathrm{U}_{3}=0$

2) Que el tratamiento 1 es igual al tratamiento 2

$\mathrm{U}_{1}-\mathrm{U}_{2}=0$

Tabla 3. Comparación de tratamientos por contrastes ortogonales 


\begin{tabular}{|l|c|c|c|c|c|c|c|c|}
\hline TRAT. & 1 & 2 & 3 & \multirow{2}{*}{$\left(\sum \mathrm{CjYj}\right) 2$} & $\sum$ njCj2 & CMError & FC & FT \\
\cline { 1 - 8 } COMP. & 9.22 & 12.74 & 15.14 & & & & \\
\hline (1 y 2) vs 3 & 1 & 1 & -2 & $(-8.32)^{2}$ & 24 & 0.078 & $36.97 *$ & 10.56 \\
\hline 1 vs 2 & 1 & -1 & 0 & $(-3.52)^{2}$ & 8 & 0.078 & $19.85^{*}$ & 10.56 \\
\hline
\end{tabular}

* Existe diferencia significativa

Fuente: de oro, Narvaez 2008.

Se puede notar que hay diferencias entre el prelimpiador 3 y los prelimpiadores 1 y 2 . Debido a esto, uno de los prelimpiadores debe ser el mejor y, de acuerdo con los datos, el 3 es el indicado para realizar los procesos de prelimpieza, descartando la posibilidad de que daría lo mismo trabajar con los otros dos. El análisis por contrastes ortogonales es claro al indicar que existen diferencias significativas entre la eficiencia de los mismos. Además, se puede afirmar que aun el prelimpiador 2 es mejor que el 1, anulando así la segunda hipótesis planteada, ya que, existen diferencias igualmente.

\section{Velocidad de llenado en prelimpiadores}

Tabla 4. Tiempo de llenado en los pre limpiadores

\begin{tabular}{|c|c|c|c|}
\hline $\begin{array}{c}\text { Característica } \\
\text { Pre limpiador }\end{array}$ & $\begin{array}{c}\text { Ángulo de } \\
\text { pendiente }\end{array}$ & $\begin{array}{c}\text { Altura de } \\
\text { descarga (cm) }\end{array}$ & $\begin{array}{c}\text { Tiempo } \\
\text { de llenado } \\
\text { (s) }\end{array}$ \\
\hline 1 & 36.86 & 10 & 20.5 \\
\hline 2 & 36.86 & 10 & 28.3 \\
\hline 3 & 24.5 & 10 & 40.8 \\
\hline
\end{tabular}

Fuente: de oro, Narvaez 2008.

A pesar de que el prelimpiador 3 presenta un tiempo de llenado mucho mayor que el de los pre limpiadores 1 y 2 , este posee una mayor velocidad de limpieza, ya que por sus dimensiones el material sólido no obstruye rápidamente los orificios presentes en las mallas limpiadoras. En fin, el tiempo de llenado no tiene una relación proporcional directa con la velocidad de prelimpieza.

\section{Características del jugo de caña recepcionado}

Tabla 5. Caracterización de jugo de caña en la recepción

\begin{tabular}{|l|c|}
\hline Característica & Valor \\
\hline Residuos sólidos $(\%)$ & 4 \\
\hline Densidad $(\mathrm{g} / \mathrm{cm} 3)$ & 1.256 \\
\hline Sólidos solubles ( ${ }^{\circ}$ Brix $)$ & 19 \\
\hline
\end{tabular}

Se puede notar que las características del jugo son normales, aunque contiene una cantidad de 
residuos sólidos considerable. Esto se debe a la no implementación de ningún tipo de mallas para filtrado y, además, a la falta de limpieza de las cañas en el momento en que se introducen en el trapiche. Este porcentaje de residuos sólidos hace necesaria la implementación de prelimpiadores para una mayor calidad de los jugos [10].

\section{Porcentaje de pre limpieza}

Tabla 6. Porcentaje de residuos sólidos recogido por los pre limpiadores

\begin{tabular}{|c|c|c|c|}
\hline Pre limpiador & $\mathbf{1}$ & $\mathbf{2}$ & $\mathbf{3}$ \\
\hline Residuos sólidos (\%) & 89.7 & 93.6 & 95.8 \\
\hline
\end{tabular}

Como se observa en la tabla, los tres pre limpiadores poseen un porcentaje de pre limpieza significativo; sin embargo, el prelimpiador 3 recoge un mayor porcentaje de residuos sólidos, básicamente porque posee dimensiones más amplias y unas mallas de filtrado de mayor área. En este caso, el porcentaje de retención de residuos sólidos es directamente proporcional a la velocidad de prelimpieza.

\section{CONCLUSIONES}

El mejoramiento de los procesos en la agroindustria panelera en Sucre sigue siendo un reto. La implementación de prelimpiadores en las estancias paneleras de veredas aledañas al municipio de San Marcos es un avance hacia la consecución de esta meta. Este trabajo deja una buena opción para la prelimpieza con resultados de hasta 3.8 litros por minuto lo que permitirá una reducción en los tiempos de residencia del jugo de caña, tanto en la clarificación como en la cocción de los mismos. Igualmente, los operarios estarán menos expuestos al calor de la hornilla, lo que generará una mejora en su salud ocupacional y un aumento en la capacidad de trabajo.

De igual modo, la producción se incrementa debido a que los tiempos de residencia disminuyen, por lo que se puede obtener una mayor cantidad del producto en menos tiempo. La caracterización de los jugos demuestra la necesidad de implementar prelimpiadores en este tipo de agroindustrias, teniendo en cuenta que la cantidad de residuos sólidos es considerable.

De otra parte, los diseños de pre limpiadores establecidos por el CIMPA son de mucha utilidad para realizar transferencia de tecnología; las medidas recomendadas son pertinentes y se pueden tener en cuenta para futuras investigaciones. La velocidad de prelimpieza es un factor determinante en el momento de elegir cuál pre limpiador será el apropiado, aunque también dependerá de la capacidad y cantidad de descarga del trapiche. Es pertinente resaltar la importancia de la Ecuación de Continuidad en este tipo de aplicaciones, debido a que su aplicación para determinar la velocidad de llenado es sencilla, práctica y efectiva, a pesar de la configuración del prelimpiador. 
En fin, el mejorar solamente el aspecto relacionado con la prelimpieza de los jugos, trae como consecuencia la optimización del proceso de manera global, puesto que se hace en un menor tiempo y se obtiene mayor cantidad de producto, sin poner en riesgo la salud de los operarios. De ahí, la importancia del trabajo y su trascendencia, en miras al crecimiento de la agroindustria panelera en la comunidad y en el departamento, aun en cualquier lugar donde se implementen este tipo de equipos.

\section{RECOMEDACIONES}

El desarrollo de un plan de capacitaciones para la comunidad, con el fin de multiplicar la implementación de pre limpiadores en las estancias del municipio San Marcos-Sucre, lo que constituye el siguiente reto en el avance de la agroindustria panelera, acompañado de Buenas Prácticas de Manufactura (BPM) para lograr un mejoramiento integral de la producción de panela.

Además, existen otros aspectos importantes, como el de realizar estudios para el mejoramiento de las hornillas ecológicas y aumentar así la velocidad de producción, utilizando la menor cantidad de leña posible.

\section{REFERENCIAS BIBLIOGRÁFICAS}

[1] CORPOICA - CIMPA. 1999 - 2000. Rendimientos básicos para el buen manejo del cultivo de caña panelera. Barbosa. Marzo de CIMPA. Diagnóstico técnico en producción y en transformación de la caña en panela de hoja en los municipios de Galeras y la subregión San Jorge. Informe Técnico (Plegable).

[2] CORPOICA - CIMPA. 2000. Caña Panelera. La Madurez y su Determinación. Mayo (Plegable).

[3] CORPOICA - CIAT.2003. Aprendiendo del pasado para proyectarnos hacia el futuro: adopción e impacto de la tecnología de panela en la Hoya del Río Suárez y Cundinamarca (Colombia).

[4] FUNDACIÓN GAVIOTA Y CÁMARA DE COMERCIO DE SINCELEJO. 2000. Investigación de mercado de las 5 líneas promisorias: lácteos, cárnicos, panela de hoja, productos agrícolas, bienes básicos de la subregión San Jorge y el municipio de Galeras, Sincelejo. Julio.

[5] INSUASTY, B. O. 1997. Variedades de caña para la producción. Memorias II Curso Internacional de Caña Panelera y su Agroindustria. CIMPA. Barbosa.

[6] MANUAL DE CAÑA PANELERA. 1992. Convenio ICA - CIMPA. Barbosa. 
[7] PRADA, L. E. 2002. Manual de mejoramiento en la calidad de miel y panela. CORPOICA - CIMPA - PRONATTA.

[8] PRADA, L. E. 1999. Cartilla buenas prácticas de manufactura en las industrias bocadillera y panelera. CORPOICA - CIMPA - SENA.

[9] RODRIGUEZ, G. 2001. Un análisis de la cadena agroindustrial. Manual de aña de azúcar para la producción de panela. CORPOICA.

[10] RODRÍGUEZ, B. G. 1997. La panela en Colombia. Un análisis de la caña agroindustrial. Memorias II Curso Internacional de Caña Panelera y su Agroindustria. CIMPA. Barbosa.

[11] RODRÍGUEZ BORRAY, Gonzalo. 2005. La Agroindustria rural de la panela en Colombia. Roles, problemática y nuevos retos.

[12] RODRÍGUEZ, B. G. 1991-2005. Cadena agroindustrial de la panela en Colombia. Una mirada desde su estructura y dinámica.

[13] SHAMES H. IRVING. 1995. Mecánica de fluidos. Mac Graw Hill 